\title{
THE INITIAL-VALUE PROBLEM IN THE QUASI-CLASSICAL THEORY OF STRONG-FIELD PHOTOIONIZATION OF RYDBERG ATOMS
}

\author{
O.V. TIKHONOVA \\ Institute of Nuclear Physics, Moscow State University \\ 119899, Moscow, Russia \\ AND M.V. Fedorov \\ General Physics Institute, Russian Academy of Sciences \\ 38 Vavilov St., 117942 Moscow, Russia
}

\begin{abstract}
Photoionization of Rydberg atoms is considered in the quasi-classical (WKB) approach. The total nonlinear strong-field ionization rate is found and investigated. The time of ionization, as a function of a growing field-strength amplitude, is shown to approach asymptotically the Kepler period $t_{\mathrm{K}}$. Interference stabilization of Rydberg atoms is confirmed to exist in the case of short pulses (shorter than the Kepler period).
\end{abstract}

PACS numbers: $32.80 . \mathrm{Fb}, 32.80 . \mathrm{Rm}$

\section{Introduction}

Interference stabilization (IS) of Rydberg atoms is known [1,2] to arise due to Rydberg-continuum $\Lambda$-type field-induced transitions between neighboring Rydberg levels. In such a case, the time of ionization $t_{\mathrm{i}}\left(\varepsilon_{0}\right)$ of a Rydberg atom in its dependence on the light field strength amplitude $\varepsilon_{0}$ was predicted $[1,2]$ to have the "death-valley" form, i.e., the form of a curve with the minimum at some critical field $\varepsilon_{\mathrm{c}}$. The quasi-classical (WKB) estimate of $\varepsilon_{\mathrm{c}}$ and $t_{\min }=t_{\mathrm{i}}\left(\varepsilon_{\mathrm{c}}\right)$ are very simple: $\varepsilon_{\mathrm{c}} \sim \omega^{5 / 3}$, where $\omega$ is the frequency of light (in atomic units) and $t_{\min } \approx t_{\mathrm{K}}=2 \pi n^{3}$, where $n$ is the principal quantum number of the originally populated Rydberg level and $t_{\mathrm{K}}$ is the classical Kepler period.

The results of the first works on IS were generalized later $[3,4]$ to take into account a possibility of excitation of Rydberg levels with higher values of the electron angular momentum $\ell$ via the $\Lambda$-type Raman transitions. The main result of such a generalization is the prediction that the "death-valley" behavior of the function $t_{\mathrm{i}}\left(\varepsilon_{0}\right)$ can be replaced by the "death-plateau" behavior which means that at $\varepsilon_{0}<\varepsilon_{\mathrm{c}}$ the function $t_{\mathrm{i}}\left(\varepsilon_{0}\right)$ falls, then at $\varepsilon_{0} \sim \varepsilon_{\mathrm{c}}$ achieves the level $\sim t_{\mathrm{K}}$, and 
remains at this level more or less constant at a rather large interval of $\varepsilon_{0}$ (such that $\varepsilon_{0} \geq \varepsilon_{\mathrm{c}}$ ).

All the above-mentioned theories of IS [1-4] were based on a series of approximations [such as the rotating wave approximation (RWA) and the "pole" approximation (PA)] validity of which is sometimes far from being evident. An alternative approach to the theory of strong-field photoionization of Rydberg atoms, free from these approximations, can be based on an attempt to apply the quasi-classical approach directly to the Schrödinger equation for a Rydberg electron in a light field $[5,6]$. In the framework of such an approach, in Ref. [5] the complex quasienergies of the system were found. However, the consideration of Ref. [5] failed to describe in a satisfactory way time evolution of the ionization probability as well as many other important characteristics of the strong-field photoionization process. For this reason, one of the conclusions of Ref. [5] consisted of the suggestion to consider in future, in the framework of the same general quasi-classical approach as in Ref. [5], the initial-value rather than the eigenvalue problem. The first attempt to realize such a program was made in our recent paper [6]. In this talk we report about our newest findings in this direction.

\section{Strong-field quasi-classical solutions of the Schrödinger equation}

The main idea of the quasi-classical approach [5-7] can be formulated as an assumption that the field-induced Rydberg-continuum transitions occur mainly in the region of electron-nucleus distances $r$ of the order of the so-called quasi-classical length $r_{\mathrm{q}}$, where [7]

$$
r_{\mathrm{q}}=\omega^{-2 / 3} \text {. }
$$

Though, typically, much larger than one $\left(r_{\mathrm{q}} \gg 1\right.$ at $\left.\omega \ll 1\right)$, the quasi-classical length is usually much shorter than the size of the Rydberg orbit $r_{\max }=2 n^{2}$, $r_{\mathrm{q}} \ll r_{\max }$. Under these conditions, the centrifugal energy in the Schrödinger energy, estimated at $r \sim r_{\mathrm{q}}$, appears to be much smaller than the Coulomb potential energy $1 / r$, if only average angular momentum is smaller than $\omega^{-1 / 3} \gg 1[7,8]$. This observation gives rise to the approximation of slow angular motion $[5,6]$, under which the centrifugal energy is dropped at all from the atomic Hamiltonian. As the result, the original three-dimensional Schrödinger equation can be reduced to the one-dimensional radial equation

$$
\mathrm{i} \frac{\partial \chi(r, t ; \theta)}{\partial t}=\left[-\frac{1}{2} \frac{\partial^{2}}{\partial r^{2}}-\frac{1}{r}+\cos (\theta) \varepsilon_{0}(t) r \sin (\omega t)\right] \chi(r, t ; \theta),
$$

where $\chi=r R$ and $R$ is the radial wave function of an electron; in a light field, both $\chi$ and $R$ depend parametrically on the angle $\theta$ between the field-strength vector $\varepsilon_{0}$ and the electron position vector; $\varepsilon_{0}(t)$ is a slow field-strength amplitude describing how a light pulse is switched on and off. Following to the ideas of Refs. [5, 6], let us solve first the Schrödinger equation (2) at a frozen angle $\theta(\theta=$ const) and, then, average the results over $\theta$. At the first stage, to simplify notations, let us drop $\cos \theta$ in Eq. (1) and further formulas keeping in mind that in the final results $\cos \theta$ has to reappear in front of $\varepsilon_{0}(t)$. 
Our goal consists of solving the initial-value problem, i.e., of finding non-perturbative solution of Eq. (2) $\chi(r, t)$ obeying the initial condition

$$
\left.\chi(r, t)\right|_{t \rightarrow-\infty}=\chi_{n}^{(0)}(r, t) \equiv \sqrt{\frac{2}{\pi n^{3} p_{n}(r)}} \sin \left\{\int_{0}^{r} p_{n}\left(r^{\prime}\right) \mathrm{d} r^{\prime}\right\} \exp \left\{-\mathrm{i} E_{n} t\right\},
$$

where $\chi_{n}^{(0)}(r, t), E_{n}=-1 / 2 n^{2}$, and $p_{n}(r)$ are the wave function, energy and quasi-classical momentum of the initially populated field-free Rydberg state,

$$
p_{n}(r)=\sqrt{2\left(E_{n}+\frac{1}{r}\right)} .
$$

By using the Euler formula for the sine on the right hand side of Eq. (3), let us present both $\chi_{n}^{(0)}(r, t)$ and $\chi(r, t)$ in the form of sums of diverging and converging waves, $\chi_{n, \pm}^{(0)}(r, t)$ and $\chi_{ \pm}(r, t)$, respectively. The initial conditions for $\chi_{ \pm}(r, t)$ have the form

$$
\left.\chi_{ \pm}(r, t)\right|_{t \rightarrow-\infty}=\frac{\mp \mathrm{i}}{\sqrt{2 \pi n^{3} p_{n}(r)}} \exp \left\{ \pm \mathrm{i} \int_{0}^{r} p_{n}\left(r^{\prime}\right) \mathrm{d} r^{\prime}-\mathrm{i} E_{n} t\right\} .
$$

Let us search for the solutions of the Schrödinger equation in the form

$$
\chi_{ \pm}(r, t)=\frac{\mp \mathrm{i}}{\sqrt{2 \pi n^{3} p_{n}(r)}} \exp \left\{ \pm \mathrm{i} \int_{0}^{r} p_{n}\left(r^{\prime}\right) \mathrm{d} r^{\prime}-\mathrm{i} E_{n} t-\mathrm{i} \sigma_{ \pm}(r, t)\right\}
$$

with the new unknown functions $\sigma_{ \pm}(r, t)$ obeying the zero initial conditions

$$
\left.\sigma_{ \pm}(r, t)\right|_{t \rightarrow-\infty}=0 \text {. }
$$

In accordance with the above-discussed role of relatively small electronnucleus distances $r \sim r_{\mathrm{q}} \ll r_{\max }$, we can expand the quasi-classical momentum $p_{n}(r)$ (4) in powers of $r\left|E_{n}\right|=r / r_{\max } \ll 1$ :

$$
p_{n}(r) \approx \sqrt{\frac{2}{r}}+E_{n} \sqrt{\frac{r}{2}} .
$$

Under this approximation and with $\chi_{ \pm}(r, t)(6)$ substituted into Eq. (2), by dropping the second-order derivative of $\sigma_{ \pm}(r, t)$ over $r$, let us reduce the arising equation for $\sigma_{ \pm}(r, t)$ to the following simplest form:

$$
\frac{\partial \sigma_{ \pm}(r, t)}{\partial t} \pm \sqrt{\frac{2}{r}} \frac{\partial \sigma_{ \pm}(r, t)}{\partial r}=\varepsilon_{0}(t) r \sin (\omega t) .
$$

The solution of this equation is easily found to be given by

$$
\begin{gathered}
\sigma_{ \pm}(r, t)=\int_{-\infty}^{t} \mathrm{~d} t^{\prime} \varepsilon_{0}\left(t^{\prime}\right) \sin \left(\omega t^{\prime}\right) r_{\mathrm{cl}}\left[ \pm t^{\prime} \mp t+\tau(r)\right] \\
\equiv \int_{0}^{\infty} \mathrm{d} t^{\prime \prime} \varepsilon_{0}\left(t-t^{\prime \prime}\right) \sin \left[\omega\left(t-t^{\prime \prime}\right)\right] r_{\mathrm{cl}}\left[t^{\prime \prime} \mp \tau(r)\right],
\end{gathered}
$$

where $t^{\prime \prime}=t-t^{\prime}$ and $\tau(r)$ is the time of motion from 0 to $r$ of a classical particle with zero total energy in the Coulomb field

$$
\tau(r)=\int_{0}^{r} \frac{\mathrm{d} r^{\prime}}{p_{n}\left(r^{\prime}\right)} \approx \frac{\sqrt{2}}{3} r^{3 / 2}
$$


$r_{\mathrm{cl}}(t)$ is the corresponding classical trajectory, or the solution of the Newton equation, or the solution of the equation $\tau\left(r_{\mathrm{cl}}\right)=t$ :

$$
r_{\mathrm{cl}}(t) \approx \frac{3^{2 / 3}}{2^{1 / 3}} t^{2 / 3}
$$

and $r_{\mathrm{cl}}(-t)=r_{\mathrm{cl}}(t)$.

By substituting $\sigma_{ \pm}(r, t)(10)$ into Eq. (6), we find the searched for solution of the Schrödinger equation (2) obeying the appropriate initial conditions (5) and (7). In its dependence on $t$, the functions $\exp [-\mathrm{i} \sigma(r, t)]$ contain a periodical part [via $\sin \left[\omega\left(t-t^{\prime \prime}\right)\right]$ in the last expression on the right hand side of Eq. (10)]. By expanding these periodical functions in the Fourier series, we get

$$
\chi(r, t)=\sum_{k} \chi_{k}(r, t) \equiv \sum_{k}\left[\chi_{k}^{+}(r, t)+\chi_{k}^{-}(r, t)\right]
$$

where $k=0, \pm 1, \pm 2, \ldots$ and the functions $\chi_{k}^{ \pm}(r, t)$ are given by

$$
\begin{aligned}
& \chi_{k}^{ \pm}(r, t)=\frac{\mp \mathrm{i} r^{1 / 4}}{2^{3 / 4} \sqrt{\pi n^{2}}} J_{k}\left[\zeta_{ \pm}(r, t)\right] \\
& \quad \times \exp \left\{ \pm \mathrm{i}\left[2^{3 / 2} \sqrt{r}+E_{n} \tau(r)\right]-\mathrm{i}\left(E_{n}+k \omega\right) t-\mathrm{i} k \phi_{ \pm}\right\} .
\end{aligned}
$$

Here $J_{k}$ are the Bessel functions, $\phi_{ \pm}$are some phases, and $\zeta_{ \pm}(r, t)$ are the functions very similar to $\sigma_{ \pm}(r, t)(10)$ :

$$
\begin{gathered}
\zeta_{ \pm}(r, t)=\left|\int_{0}^{\infty} \mathrm{d} t^{\prime \prime} \varepsilon_{0}\left(t-t^{\prime \prime}\right) \exp \left(\mathrm{i} \omega t^{\prime \prime}\right) r_{\mathrm{cl}}\left[t^{\prime \prime} \mp \tau(r)\right]\right| \\
\equiv\left|\int_{-\infty}^{t} \mathrm{~d} t^{\prime} \varepsilon_{0}\left(t^{\prime}\right) \exp \left(-\mathrm{i} \omega t^{\prime}\right) r_{\mathrm{cl}}\left[t-t^{\prime} \mp \tau(r)\right]\right| .
\end{gathered}
$$

In a general form, Eqs. (13)-(15) solve the problem formulated in the beginning of this section: they determine the solution of the Schrödinger equation obeying the initial condition (3).

\section{Above-threshold and total ionization}

The quickest variations of the functions $\chi_{k}^{ \pm}(r, t)(13)$ in time $t$ are determined by the factors $\exp \left[-\mathrm{i}\left(E_{n}+k \omega\right) t\right]$. For this reason, the functions $\chi_{k}(r, t)$ can be interpreted as the wave functions of the above-threshold wave packets with mean energies equal to $E_{n}+k \omega$. The $t$ - and $r$-dependent electron density in the $k$-th above-threshold wave packet is given by

$$
\rho_{k}(r, t)=\left|\chi_{k}(r, t)\right|^{2}=\left|\chi_{k}^{+}(r, t)+\chi_{k}^{-}(r, t)\right|^{2} .
$$

The total probability of ionization to the time $t$ can be determined as

$$
w_{i}(t)=\sum_{k=1}^{\infty} \int_{0}^{\infty} \mathrm{d} r \rho_{k}(r, t)=\sum_{k=1}^{\infty} \int_{0}^{\infty} \mathrm{d} r\left|\chi_{k}^{+}(r, t)+\chi_{k}^{-}(r, t)\right|^{2} .
$$

Let us discuss now the $r$-dependence of the functions $\chi_{k}^{ \pm}(r, t)(14)$. The quickest part of this dependence is determined by the factor $\exp \left( \pm \mathrm{i} 2^{3 / 2} \sqrt{r}\right)$. This dependence disappears in the squared absolute values of the functions $\chi_{k}^{ \pm}(r, t)$. But 
in their cross-product, $2 \operatorname{Re}\left\{\left[\chi_{k}^{+}(r, t)\right]^{*} \chi_{k}^{-}(r, t)\right\} \propto \cos \left(2^{5 / 2} \sqrt{r}\right)$, this dependence gives rise to very fast oscillations with a period of the order of one in atomic units. Being averaged over these fast oscillations, Eq. (17) gives

$$
\begin{aligned}
w_{i}(t) & =\sum_{k=1}^{\infty} \int_{0}^{\infty} \mathrm{d} r\left(\left|\chi_{k}^{+}(r, t)\right|^{2}+\left|\chi_{k}^{-}(r, t)\right|^{2}\right) \\
& =\int_{0}^{\infty} \frac{\mathrm{d} r \sqrt{r}}{4 \sqrt{2} \pi n^{3}}\left\{2-J_{0}^{2}\left[\zeta_{+}(r, t)\right]-J_{0}^{2}\left[\zeta_{-}(r, t)\right]\right\} .
\end{aligned}
$$

Let us discuss now the structure of the functions $\zeta_{ \pm}(r, t)(15)$ determining the arguments of the Bessel functions in Eqs. (14) and (18). In accordance with the definition (15), these functions are given by the integrals of products of the fast oscillating $\left[\exp \left(-\mathrm{i} \omega t^{\prime}\right)\right]$ and slow $\left(\varepsilon_{0}\left(t^{\prime}\right) r_{\mathrm{cl}}\left[t-t^{\prime} \mp \tau(r)\right]\right)$ functions. Such integrals are known to be determined mainly by the ends of the integration regions, if only the slow part of the integrand is everywhere smooth. An important part of these slow function is the function $r_{\mathrm{cl}}\left[t-t^{\prime} \mp \tau(r)\right]$ in which $t^{\prime} \leq t$ and $r(r) \geq 0$. For the lower sign the argument of $r_{\mathrm{cl}}$ is always positive, whereas for the upper sign it changes sign at $t^{\prime}=t-\tau(r)$. In terms of the corresponding classical trajectories this means that in the first case the classical particle comes monotonously from infinity to the point $r$, whereas in the second case (the upper sign in the argument of $r_{\mathrm{cl}}$ ) it comes from infinity, reaches the origin $r=0$, experiences reflection, and then returns to the point $r$ (see Fig. 1). In fact it appears that just this reflection from the origin is responsible for irreversible ionization. Mathematically, the reflection of the classical trajectory from the origin means that, in the case of $\zeta_{+}$, the slow part of the integrand has a cusp at the point $t^{\prime}=t-\tau(r)$. Hence, in this case

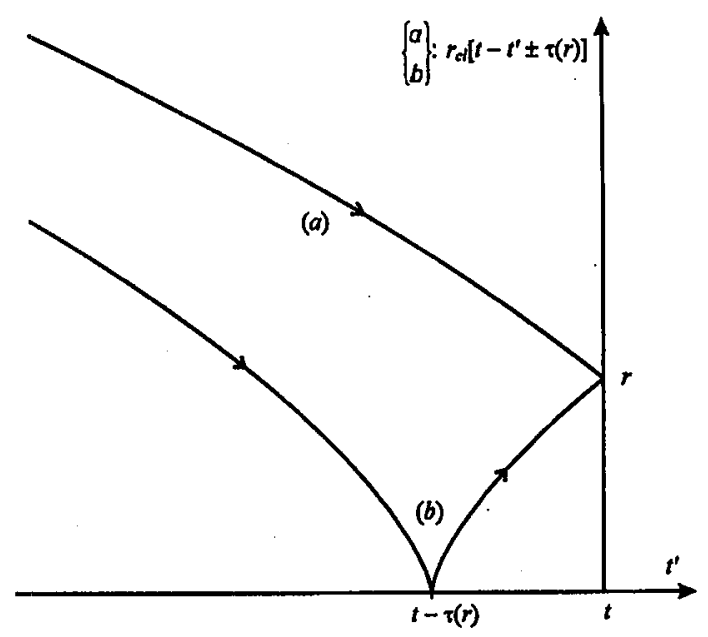

Fig. 1. The classical trajectories $r_{\mathrm{cl}}\left[t-t^{\prime}+\tau(r)\right]$ (a) and $r_{\mathrm{cl}}\left[t-t^{\prime}-\tau(r)\right]$ (b) determining the functions $\zeta_{-}(r, t)$ and $\zeta_{+}(r, t)(15)$, respectively. The arrows indicate the direction of motion over the classical trajectories. 
the integral over $\mathrm{d} t^{\prime}$ from $-\infty$ to $t$ turns in fact in the sum of two integrals with different integrands, from $-\infty$ to $t-\tau(r)$ and from $t-\tau(r)$ to $t$. This is the limit $t^{\prime}=t-\tau(r)$ in both of these two integrals that gives the main contribution to the irreversible ionization. Not dwelling upon any further details of calculations, let us reproduce the result of integration in (15) for the case of large $t, t \gg T$, where $T$ is the pulse duration, i.e., for the case when the light pulse has gone

$$
\zeta_{+}(r, t)=\frac{2^{2 / 3} 3^{1 / 6} \Gamma\left(\frac{2}{2}\right) \varepsilon_{0}[t-\tau(r)]}{\omega^{5 / 3}}, \quad \zeta_{-}(r, t)=0
$$

where $\Gamma(x)$ denotes the gamma-function.

Equation (19) describes the wave packet that moves away from the nucleus. The shape of this packet coincides with that of the pulse envelope. With the help of Eq. (19) the general expression (18) for the probability of ionization per pulse $w_{\mathrm{i}}$ can be further simplified. The substitution of the integration variable $r$ by $\tau(r)$ and, then, by $t$ gives

$$
w_{\mathrm{i}}=\int_{-\infty}^{\infty} \frac{\mathrm{d} t}{2 t_{\mathrm{K}}}\left\{1-J_{0}^{2}\left[\frac{2^{2 / 3} 3^{1 / 6} \Gamma\left(\frac{2}{3}\right) \varepsilon_{0}[t-\tau(r)]}{\omega^{5 / 3}}\right]\right\}
$$

where $t_{\mathrm{K}}=2 \pi n^{3}$ is the Kepler period.

Finally, for a square pulse of a duration $T$ Eq. (20) yields

$$
w_{\mathrm{i}}=\Gamma T
$$

where $\Gamma$ is the nonlinear rate of transitions

where

$$
\Gamma=\frac{1}{2 t_{\mathrm{K}}}\left[1-J_{0}^{2}(\zeta)\right]
$$

$$
\zeta=\frac{2^{2 / 3} 3^{1 / 6} \Gamma\left(\frac{2}{3}\right) \varepsilon_{0}}{\omega^{5 / 3}}
$$

and now $\varepsilon_{0}=$ const. In the weak-field limit $\left(\varepsilon_{0} \ll \omega^{5 / 3}\right)$, Eq. (22) yields the Fermi-golden-rule rate of ionization

$$
\Gamma_{\mathrm{FGR}}=\frac{3^{1 / 3} \Gamma\left(\frac{2}{3}\right) \varepsilon_{0}^{2}}{2^{5 / 3} \pi n^{3} \omega^{10 / 3}}\left|\left\langle Y_{l^{\prime}, m}|\cos \theta| Y_{l, m}\right\rangle\right|^{2},
$$

where $Y_{l, m}$ are spherical functions, $l$ and $l^{\prime}$ denote the electron angular mornentum in the initial and final states, $m$ is $z$-projection of the angular momentum, and the factor $\left|\left\langle Y_{l \prime}^{\prime \prime}, m|\cos \theta| Y_{l, m}\right\rangle\right|^{2}$ is added to the expression following from Eqs. (22) and (23) to take into account the electron angular motion. In the case of a strong field, $\varepsilon_{0} \geq \omega^{5 / 3}$, the angular motion of a Rydberg electron can hardly be taken into account rigorously in such a simple way. However, in the approximation of a slow angular motion discussed in the beginning of Sec. 2 we can use the procedure of Ref. [5]. In the framework of this procedure, in the expressions (22) and (23) for the strong-field rate of ionization, the field strength amplitude $\varepsilon_{0}$ has to be substituted by $\varepsilon_{0} \cos \theta$ and the result has to be averaged over $x \equiv \cos \theta$ to give

$$
\bar{\Gamma}=\frac{1}{2 t_{\mathrm{K}}}\left[1-\int_{0}^{1} \mathrm{~d} x J_{0}^{2}(\zeta x)\right] .
$$

The characteristic time of ionization can be determined as the inverse double rate of ionization 


$$
t_{\mathrm{i}}=1 / 2 \Gamma \quad \text { and } \quad \bar{t}_{\mathrm{i}}=1 / 2 \bar{\Gamma}
$$

for the case $\theta=0$ and for the rate $\bar{\Gamma}(25)$ averaged over $\theta$, respectively.

\section{Discussion}

The dependence of the ionization rates and time of ionization on the field-strength parameter $\zeta(23)$ is shown in Figs. 2 and 3 for the two above-discussed cases: for $\theta=0$ (Fig. 2) and for $\bar{\Gamma}$ (25) averaged over $\theta$ (Fig. 3), the rates of ionization and the time of ionization are measured in units of the inverse double Kepler period and Kepler period, respectively. The pictures of Fig. 2 show that for a given $\theta$, both the rate and time of ionization are oscillating functions of the field-strength amplitude $\varepsilon_{0}$. This result agrees with and specifies the qualitative prediction of Ref. [5]. By comparing the results of the present theory with those of Refs. [1-4], we can make an assumption that, possibly, the oscillating dependencies $t_{\mathrm{i}}\left(\varepsilon_{0}\right)$ and $\Gamma\left(\varepsilon_{0}\right)$ arise when and because one does not use the rotating-wave and pole approximations inherently present in the earlier theories [1-4]. The minima of the oscillating curve $t_{\mathrm{i}}(\zeta)$ and the maxima of the curve $\Gamma(\zeta)$ correspond to the

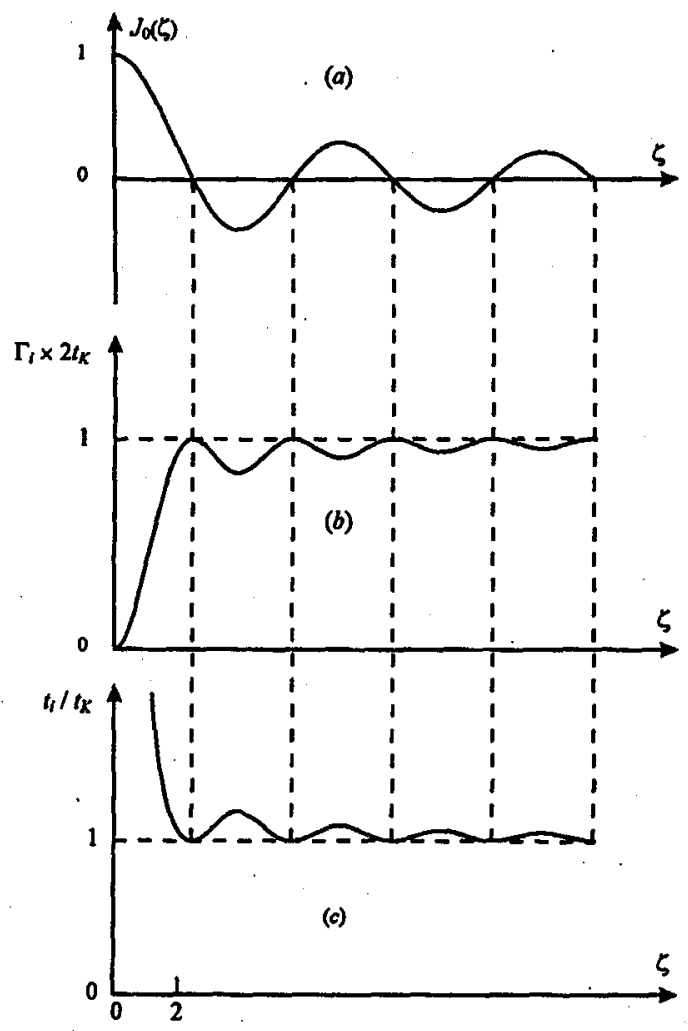

Fig. 2. The zero-order Bessel function (a), the rate of ionization $\Gamma$ (b), and the time of ionization $t_{\mathrm{i}}$ (c) vs. the field-strength parameter $\zeta(23)$. 
positions of zeros of the zero-order Bessel function $J_{0}(\zeta)$ shown for comparison in Fig. 2a. The minimal time of ionization achieved at these points is equal to one Kepler period $t_{\mathrm{K}}$ and the corresponding maximal rate of ionization is equal to $1 /\left(2 t_{\mathrm{K}}\right)$. This means that the strong-field stabilization in Rydberg atoms has to occur if the pulse duration is less or of the order of the Kepler period, in accordance with the main ideas of Refs. [3, 4]. In agreement with Refs. [3, 4], the "death-plateau" rather than the "death-valley" behavior of the curve $t_{\mathrm{i}}\left(\varepsilon_{0}\right)$ is confirmed: in the strong-field limit the function $t_{\mathrm{i}}\left(\varepsilon_{0}\right)$ saturates at the level $t_{\mathrm{K}}$ rather than grows unlimitedly.

The curves of Fig. 3 show that the above-discussed oscillations appear to be smoothed out when the electron angular motion is taken into account, though in the framework of a very rough and approximate procedure of averaging over $\theta$. This result can be interpreted as an indication that the $3 \mathrm{D}$ structure of an atom can result in a well pronounced "death-plateau" structure of the dependence $t_{\mathrm{i}}\left(\varepsilon_{0}\right)$ : saturation in a strong field at the level $t_{\mathrm{K}}$ without any oscillations or growth. This conclusion agrees with that of Refs. $[3,4]$ though the methods of analysis in these papers were absolutely different from that of the present one.

At last, it should be mentioned that, in the case of rectangular pulses, the derived probability of ionization per pulse (21) depends linearly on the pulse duration $T$, though this is not the lowest order perturbation theory and the rate of ioniza-

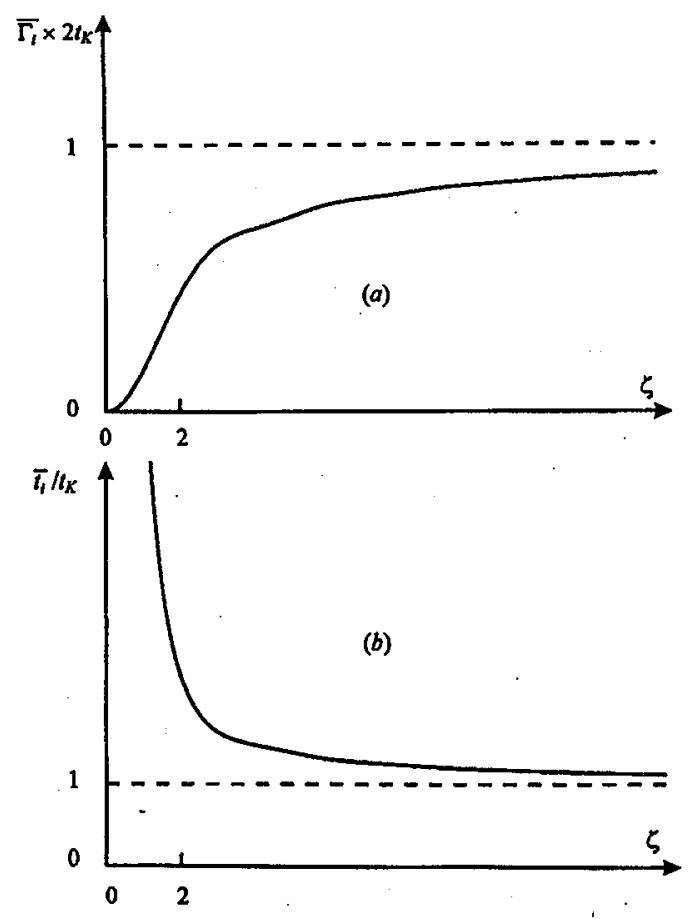

Fig. 3. The rate of ionization averaged over $\theta, \bar{\Gamma}_{\mathrm{i}}$ (a) and the corresponding "average" time of ionization $\bar{t}_{\mathrm{i}}=1 /\left(2 \bar{\Gamma}_{\mathrm{i}}\right)(\mathrm{b})$ vs. $\zeta(23)$. 
tion $\Gamma(22)$ is not a linear function of $\varepsilon_{0}^{2}$. The linear dependence of the ionization probability $w_{\mathrm{i}}$ on the pulse duration $T$ means that the suggested theory cannot describe the regime of depletion of the initially populated Rydberg state. Equation (21) assumes that the results derived are valid only if $\Gamma T<1$, i.e., only for short pulses. In the strong-field case, when $\Gamma \sim 1 / t_{\mathrm{K}}$, this limitation yields $t<t_{\mathrm{K}}$, i.e., the pulse duration has to be shorter than the Kepler period. These restrictions are explained by the used approximations in which the quasi-classical momentum $p_{n}(r)(4)$ was expanded in powers of $r\left|E_{n}\right| \ll 1(8)$ and the squared derivative of the functions $\sigma_{ \pm}(r, t)$ was dropped in Eq. (9). Unfortunately, these approximations are crucially important for the found above rather simple solutions of the Schrödinger equation (13)-(15) to be valid. Construction of a theory free from the discussed approximations can be very interesting but also much more complicated than in the case considered here. Another equally important but, probably, equally difficult direction of future investigations has to include attempts to consider more rigorously the electron angular motion. We hope to return to these problems later.

\section{Acknowledgment}

The work is supported partly by the Russian Fund of Basic Research (the grants \#\# 96-02-19286 and 96-02-17649), and by the Civilian Research and Development Fund (USA) jointly with the Russian State Committee on Science and Technologies (the grant \# RP1-244).

\section{References}

[1] M.V. Fedorov, A.M. Movsesian, J. Phys. B 21, L155 (1988).

[2] M.V. Fedorov, Laser Phys. 3, 219 (1993).

[3] M.V. Fedorov, M.-M. Tegranchi, S.M. Fedorov, J. Phys. B 29, 2907 (1996).

[4] A. Woiczik, R. Parzynski, Laser Phys. 7, 574 (1997).

[5] M.V. Fedorov, J. P.hys. B 27, 4145 (1994).

[6] O.V. Tikhonova, M.V. Fedorov, Laser Phys. 7, 574 (1997).

[7] M. Adams, M.V. Fedorov, V.P. Krainov, D.D. Meyerhofer, Phys. Rev. A 52, 125 (1995).

[8] N.B. Delone, S.P. Goreslavsky, V.P. Krainov, J. Phys. B 16, 2369 (1983); 22,

2941 (1989). 